\title{
The behaviour of a low voltage distribution network with crescent presence of photovoltaic generation and energy storage elements
}

\author{
Rafael Martins Leite, Jonas Villela de Souza, Eduardo Nobuhiro Asada and Mário Oleskovicz \\ Department of Electrical and Computer Engineering \\ São Carlos Engineering School, University of São Paulo \\ São Carlos, Brazil \\ E-mail: rafaelleite@usp.br; jonas.villela@usp.br; easada@usp.br; and olesk@sc.usp.br.
}

\begin{abstract}
This paper aims to examine the increasing integration of distributed energy resources in the low voltage distribution grid. Considering the current increasing of distributed photovoltaic generation in Brazil, there is a deterioration of specific indices related to power quality, such as overvoltage and voltage unbalance. In this sense, a survey of these problems, the severity assessment via simulation of a real system and the proposal of improvement in the associated indexes using energy storage devices are made. The resulting analyses contemplate both the current reality of growth in the distributed photovoltaic generation and the joint integration of these generators with energy storage systems, thus enabling a comparison of results focusing on the electricity grid impacts. The study has improved the network's operation, reducing the power quality aggravating factors and increasing the local consumption of the energy generated locally. The total losses on the original network were reduced by up to $52.6 \%$, the voltage drop to $74.7 \%$ and the voltage rise was completely eliminated.
\end{abstract}

Keywords. Power quality; Energy storage system; Distributed energy resources; Distributed photovoltaic generation; Low voltage distribution grid.

\section{Introduction}

Since 1987, with the Montreal Protocol, the global energy sources' transition has become a priority. In the last decade, renewable-based power generation's capacity has practically doubled, increasing from $1,227 \mathrm{GW}$ to 2,537 $\mathrm{GW}$ [1]. In the same period, the renewable source with the most significant increase was the photovoltaic (PV). In 2019 , it surpassed the $580 \mathrm{GW}$ mark in its installed capacity worldwide, representing a 14-fold growth since 2010 [1]. According to the Brazilian Photovoltaic Solar Energy Association [2], from 2012 to August 2020, the country had installed a capacity of $6,4 \mathrm{GW}$, of which $53 \%$ corresponds to Distributed Generation (DG).

However, PV generation brings some technical challenges regarding its assimilation into the electrical system. Due to its sunlight-dependent operation, the production is restricted to the daytime period. Such characteristic causes a mismatch between the generation and local consumption curves (Paixão et al., 2018). It results in a bidirectional power flow throughout the day, which can cause unexpected increases in voltage levels and irregular device operations and protection philosophies [4]. Due to climatic changes and shading caused by clouds, an inconstant generation is often obtained, which can result negatively in the Power Quality (PQ) indicators [5].

In order to mitigate such challenges, energy storage technologies have been an option. The Energy Storage System (ESS) has been a well-established resource for reducing peak loads. By storing energy in periods of low demand, the ESS can supply the demand in a decentralized manner in moments of high consumption, thus reducing the power flow that leaves the substation [6]. Introducing this strategy into the DG context, storage can be done when there is a local generation surplus in proportion to consumption. This stored energy will make it possible to supply the demand in its peak moments, which usually occurs at the end of the day, considering a residential profile [7].

The purpose of this paper is to verify how a Low Voltage Distribution Network (LVDN) reacts to the current growth model of Distributed Photovoltaic Generators (DG-PV). Subsequently, following commercial indicators, the ESS is added to the DG-PV, and thus, the possible impacts are reassessed. Such an association of Distributed Energy Resources (DER) proved to be effective in reducing the demand for energy in the LVDN, relieving the electricity grid at peak consumption while also reducing the percentage levels of overvoltage and voltage unbalance. Finally, there was also a considerable reduction in active power losses in the line conductors and the transformer.

This work is divided into five sections, the first one being this introduction. The Section 2 provides a brief description of the limitations in the integration of DG-PV in the network. Section 3 presents the materials and methods used in the simulations. In Section 4 the results are exposed and discussed. The conclusions are drawn in Section 5. 


\section{Challenges Description}

Since PV power has an intermittent characteristic, with a generation being restricted to a certain period of the day, it may not completely meet the customers' power demand. With the popularization and the increasing insertion of PV in homes and small businesses, the direction of power flow throughout the day is expected to undergo substantial changes. Therefore, the focus in this paper is on effects resulting from the relationship between consumption and generation profiles.

\section{A. Load Profiles}

From the distributor's perspective, a residence is a variable load with a daily behaviour profile. It is explained since electrical appliances are directly related to the people who use them daily.

In the current scenario, the Prosumer figure appears when consumers install power generation to satisfy their needs and also to be able to inject energy into the network. So it is necessary to analyse its behaviour in the context of residential consumption, as illustrated in power profile shown in Figure 1. To generate an amount of energy equivalent to the installed load, DG-PV may reach higher active power levels and generation surplus which is injected into the network, may exist. In this context, the resulting curve can assume negative values, indicating a reverse active power flow at the connection point. Normally, the peak of consumption in the evening, characteristic of household consumption, persists.

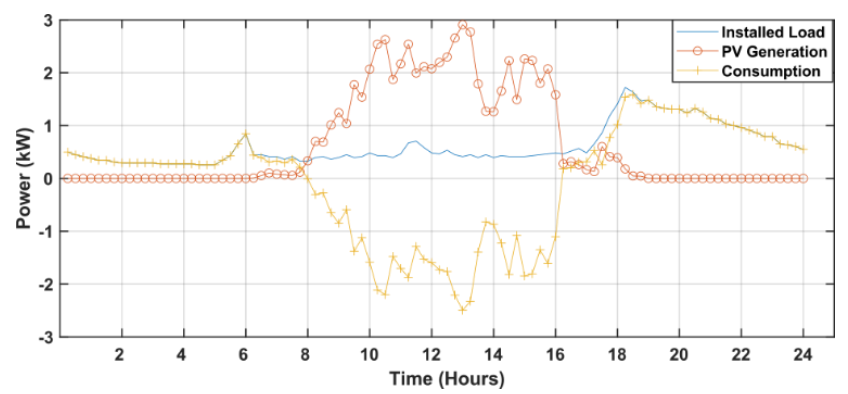

Figure 1 - Demand profile of a Prosumer with DG-PV.

If this tendency is not observed and planned by the electric utilities, it may suffer economic losses related to the purchase and sale of energy. Next to that, the network's operation must be restructured according to this new reality to avoid efficiency loss, voltage deviations in the feeder's terminals, and phase unbalance.

\section{B. Variation of Voltage Levels}

Torquato et al. (2018) have shown that the most significant operational limitation for hosting the DG-PV is the overvoltage caused at its coupling point. The Brazilian regulator states that installations up to $1 \mathrm{kV}$ must remain in a range of $92 \%$ to $105 \%$ of their nominal voltage value for at least $97 \%$ of the time [9]. Taking a Prosumer's Common Coupling Point (PCCP) as a reference, assuming that the DG-PV has a unitary power factor and that its generation is greater than consumption at a particular moment, one can assess how such an effect arises. Such a situation is shown in Figure 2.

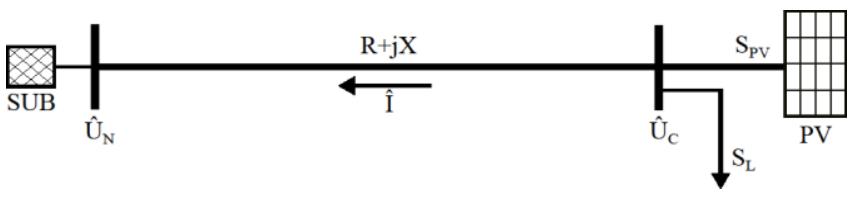

Figure 2 - Simplified model of the DG-PV 's connection to the distribution system.

With the inversion of the power flow in contrast to what would be usual, there will be an effect of increasing the PCCP voltage concerning the network reference. By Ohm's Law and the relationships between voltage, current, and power, the voltage is obtained in the Prosumer's PCCP, given by Equation 1 [10].

$$
\mathbf{U}_{\mathbf{c}}=\hat{\mathbf{U}}_{\mathbf{N}}+\mathbf{R}\left(\frac{\mathbf{P}+\mathbf{Q}\left(\frac{\mathbf{X}}{\overline{\mathbf{R}}}\right)}{\mathbf{U}_{\mathbf{c}}}\right)+\mathbf{j R}\left(\frac{\mathbf{P}\left(\frac{\mathbf{X}}{\overline{\mathbf{R}}}\right)-\mathbf{Q}}{\mathbf{U}_{\mathbf{c}}}\right)
$$

Considering that the DG-PV has a fixed and unitary power factor (pf) and that the reactive consumption in residence is low, the complex part resulting from the power will also be small. The $\mathrm{X} / \mathrm{R}$ ratio, on the other hand, is usually less than 1 for short grids [10]. Therefore, it is reasonable to disregard the voltage variation in quadrature. Henceforth, it is possible to affirm that the injection of active power in the network is responsible for increasing voltage in the network, as indicated by Equation 2:

$$
\Delta \boldsymbol{U}=\boldsymbol{R}\left(\frac{\boldsymbol{P}}{\boldsymbol{U}_{c}}\right)
$$

\section{Voltage Unbalance}

Voltage unbalance is a condition in which the three-phase voltages differ in amplitude or are displaced from their normal $120^{\circ}$ phase relationship or both. ANEEL (Brazilian National Electric Energy Agency) determines that for systems up to $1 \mathrm{kV}$, the percentage imbalance index must be less than 3\%. It is established that this limit is respected in at least $95 \%$ of the 1,008 valid voltage measurements [9]. This Voltage Unbalance Factor (VUF\%) is the percentage ratio between the negative sequence voltage $\left(\boldsymbol{V}^{-}\right)$and the positive $\left(\boldsymbol{V}^{+}\right)$.

Considering that the change in power flow caused by the DG-PV influences voltage levels and that many of these generators are single-phase or two-phase, the level of unbalance network may become a problem with increased penetration of DG-PV. If load and power injection are not evenly distributed between the three phases, their amplitude and phase angle may change, thereby increasing the level of unbalance and possibly exceeding the regulatory agency's limits. 


\section{Analysis Method}

Firstly, the consumption profiles smoothing, combining residential DG-PV with the application of ESS (represented by a battery bank) is proposed. With the load profile forecast and adequate ESS control, the aim is to balance periods of high generation and consumption, ensuring that the surplus of energy generated can be used at a more opportune moment. In this way, this surplus is prevented from being injected into the network and causing the effects already mentioned.

The simulations that make up this study are divided into three steps. In the first step, a test distribution system is analysed. During the second step, the presence of DG-PV is progressively increased until all consumers behave as Prosumers. In the third step, distributed ESSs are added and allocated to each Prosumer. Still in the third step, distributed storage units are disregarded, which will give place to a single and concentrated ESS located at the beginning of the feeder.

Essentially, each of these steps has a specific purpose. First of all, it is necessary to evaluate the original test system's performance, enabling a comparison parameter for all other cases. Then, the impacts of the continuous integration of DG-PV in the LVDN are studied. To mitigate the possible problems caused by the previous scenario, ESSs are inserted as individual DER in each residential installation. Finally, the possibility of a larger concentrated ESS that handles the entire network in a unified manner is also considered.

\section{A. The Test system}

The low voltage test system used has 41 buses and is shown in Figure 3. It is characterized as an LVDN located in Brazil's southeaster region, which feeds 34 consumers through a three-phase $45 \mathrm{kVA}$ delta-star transformer with a grounded neutral [10]. The respective network was modelled using OpenDSS software [11]. The voltage level of the network is set to 1 p.u.

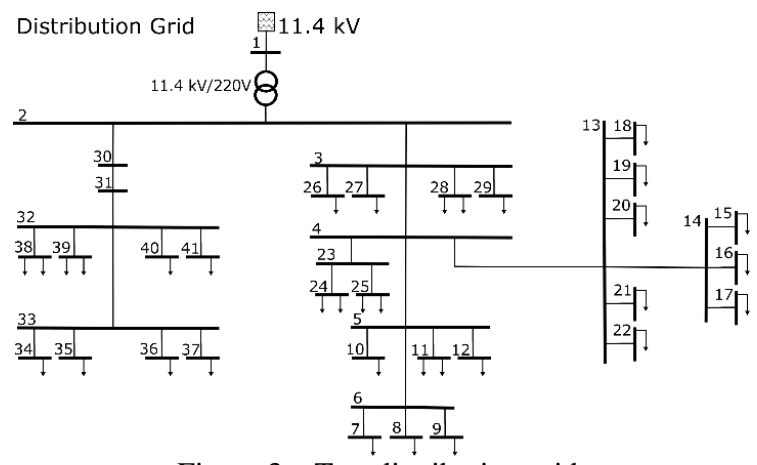

Figure 3 - Test distribution grid.

Each load is considered to have a nominal active power and a pf of 0.85 and is also associated with a load curve, representing the daily variation of consumption in p.u., sampled at 15-minute intervals, totalling 96 points. The load curve is based on the maximum consumption value assigned to each residence.

\section{B. $G D-P V$}

The used PV model is described in the OpenDSS PVSystem and InvControl Element Models Manual [12]. The generator consists of the solar panels' arrangement and an AC-DC inverter, represented by a current source connected to the grid.

Each generator has the maximum rated generation power, efficiency, and pf as main attributes. Then, each DG-PV power that will be connected to the test system is calculated by the so-called "commercial method." The pf used for all photovoltaic systems is unitary since there is the only generation of active power. Operation is defined from two inputs: solar irradiance and temperature. These are daily curves, considering a sunny summer day.

The most common commercial method for dimensioning the DG-PV considers the average daily consumption, which can be obtained from the annual history present in the interested consumer's energy bill. The calculation consists of dividing the average daily consumption by the generation potential of the location where the generator will be installed. In this way, the nominal power of each DG-PV is obtained.

Similarly, the simulations' daily load $\left(\mathbf{P}_{\mathbf{c}}(\mathbf{t})\right)$ and irradiation $(\operatorname{Ir}(\mathbf{t}))$ curves are integrated. The former is then divided by the latter, and, finally, the result is multiplied by the average daily PV efficiency $\left(\boldsymbol{\eta}_{\mathbf{p v}}\right)$.

\section{PV Penetration}

The most common practice to define the DG penetration level is to compare the installed generators' nominal powers with that of the transformer responsible for supplying the grid [13]. Based on the method presented in the previous item, the DG-PV was then calculated for each of the 34 consumers connected to the test system, who become Prosumers. Subsequently, the percentage level of the nominal PV power was calculated from the $45 \mathrm{kVA}$ distribution transformer's capacity.

The increase in DG-PV followed a decreasing logic concerning daily energy consumption, as seen in Figure 4. This scenario is acceptable since the higher the energy consumption, the greater the benefit of installing the GD$\mathrm{PV}$ and the greater the financial interest. For the system in question, the maximum penetration percentage is $144 \%$.

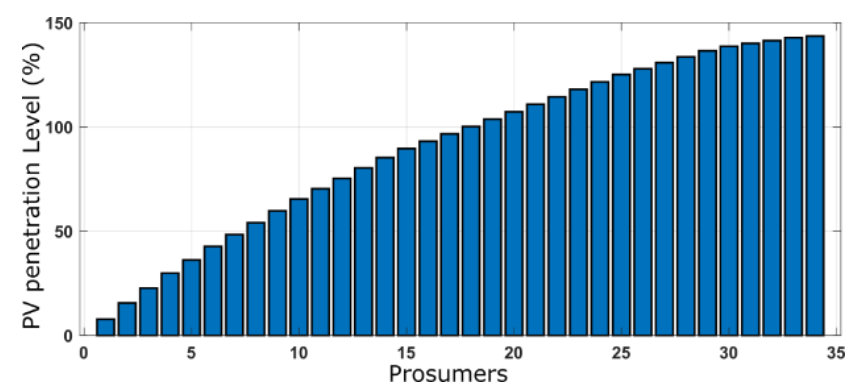

Figure 4 - Increasing of DG-PV nominal power in the grid 


\section{Storage}

The ESS model used is the standard OpenDSS model. Besides, its detailed description is made by Rocha \& Radatz (2020). The system consists of an ideal ESS, a bidirectional inverter, and elements representing the losses associated with charge, discharge, and inactivity.

To define each ESS's capacity to be installed, the difference between the active powers of generation and load consumption during the DG-PV activity period (8 am to $4 \mathrm{pm}$ ) was considered. Thus, it is guaranteed that all excess energy is reserved in the ESS and not injected into the network. The ESS capacity's calculated values were approximate to the same ones described in an Ion-lithium batteries manufacturer's datasheet. The data on the rated power and discharge depth were also obtained (RESU, 2016).

\section{E. ESS Control Mode}

Logic was created to control each storage states (idling, charging, and discharging) and its power $\left(\mathbf{P}_{\mathbf{E S S}}(\mathbf{t}+\mathbf{1})\right)$. For this purpose, the instantaneous power consumption is measured at the PCCP $\left(\mathbf{P}_{\mathbf{L}}(\mathbf{t})\right)$. A lower limit $\left(\mathbf{L}_{\mathbf{l}}\right)$ and a superior limit $\left(\mathbf{U}_{\mathbf{l}}\right)$ were also set, where it is desired that the energy demand of the grid remains. Such logic was based on a peak load shaving strategy [15].

To avoid oscillations caused by sudden variations in the DG-PV, the $\mathbf{P}_{\text {follow }}$ curve is applied for charging. This curve results from the profiles associated with the system (local consumption and generation). The logic used is represented by Equations 3 and 4:

$$
\begin{aligned}
& \mathbf{P}_{\text {follow }}(\boldsymbol{t})=\mathbf{P}_{\mathbf{c}}(\boldsymbol{t})-\mathbf{P}_{\mathrm{pv}}(\boldsymbol{t}) \\
& \mathbf{P}_{\mathrm{ESS}}(\mathbf{t}+\mathbf{1})=\left\{\begin{array}{cc}
\mathbf{P}_{\text {follow }}(\mathbf{t}+\mathbf{1}), & \text { se } \mathbf{P}_{\mathbf{L}}(\mathbf{t})<\mathbf{L}_{\mathbf{l}}(\text { charge }) \\
0, & \text { se } U_{1} \leq \mathbf{P}_{\mathbf{L}}(\mathbf{t}) \geq \mathbf{L}_{\mathbf{l}}(\text { idling }) \\
\mathbf{P}_{\mathbf{L}}(\mathbf{t})-\mathbf{U}_{1}, & \text { se } \mathbf{P}_{\mathbf{L}}(\mathbf{t}) \geq \mathbf{U}_{\mathbf{l}}(\text { discharge })
\end{array}\right.
\end{aligned}
$$

For the ESS to store all surplus energy generated locally, $\mathbf{L}_{\mathbf{l}}$ was set to 0 (zero). On the other hand, to reduce peak consumption in the late afternoon $\mathbf{U}_{\mathbf{l}}$ was set at $30 \%$ of the maximum nominal load value for each Prosumer.

\section{Discussion on the Results}

Prosumers, consumers who once were passive and only received the energy made available by the utility company, can now supply part of their demand and still inject the excess of their production into the network. Figure 5 shows the active power measured at the transformer's secondary for seven different DG-PV penetrations.

Under analysis, phase $B$ presents the most considerable voltage magnitudes variations in the test system since it is the most loaded. Figures 6 and 7 show the voltage values in p.u. of the original network $(0 \%)$ and with the maximum penetration $(144 \%)$ in phase $B$. The voltage values were almost always close to the nominal value on the original network. Now they present a substantial increase, reaching $3.92 \%$. However, it is also noted that the voltage drop resulting from increased consumption during peak hours remains.

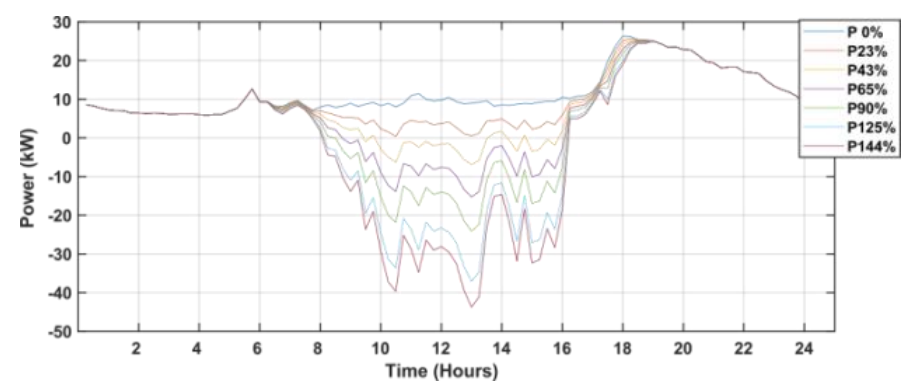

Figure 5 - Active power on the transformer's low voltage side for different penetration levels of the DG-PV.

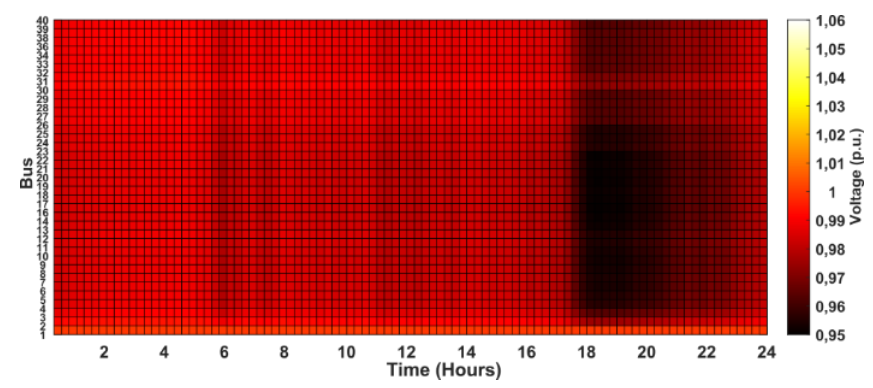

Figure 6 - Bus voltage variations throughout the day without

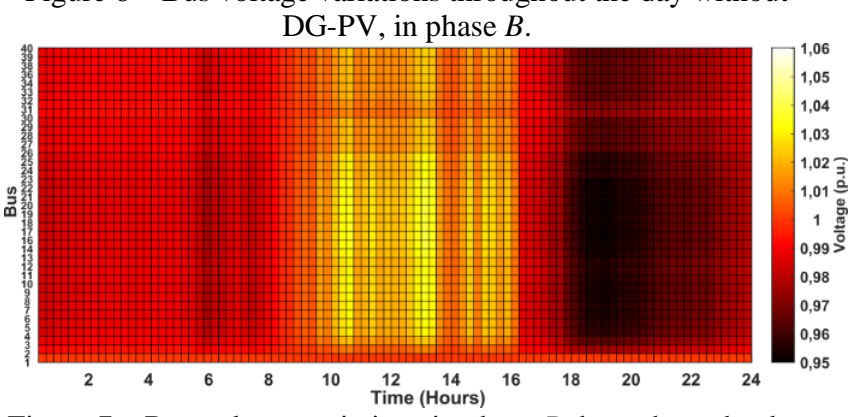

Figure 7 - Bus voltage variations in phase $B$ throughout the day with $144 \%$ DG-PV penetration.

Figure 8, as Figure 3, shows the measurements of active power during 24 hours for the same levels of penetration for the associations of DG-PV-ESS. In this scenario, the lower $\left(\mathbf{L}_{\mathbf{l}}\right)$ and upper $\left(\mathbf{U}_{\mathbf{l}}\right)$ limits (Equation 4) for each ESS are defined as 0 (zero) and $30 \%$ and all storage simulations are started with minimal loading.

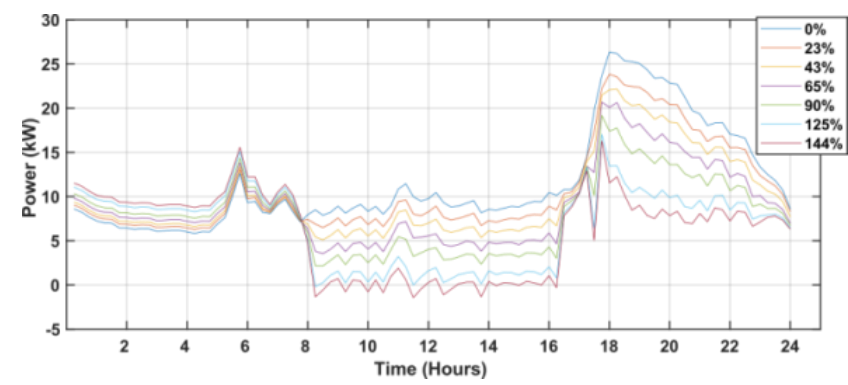

Figure 8 - Active power on the transformer's low voltage side for different penetration levels of the DG-PV-ESS.

The first point to note is the different demands for active power in the morning, before the start of PV generation and the subsequent loading of the ESSs. Until their load begins to increase, all ESSs will be inactive once they start with their minimum reserve. 
Whereas each storage unit has been modelled to absorb all the difference between local consumption and generation, there will no longer be a surplus of power to be injected from the primary to the secondary network. This way, what results is a gradual decrease in demand for each DERs penetration until it is very close to zero. Even so, there are still some fluctuations between positive and negative values in the maximum DG-PV-ESS penetration.

Finally, the peak consumption present in the late afternoon and early evening is deficient with integrating the DERs combination. The highest value recorded in the original system at $6 \mathrm{pm}$ is $26.35 \mathrm{~kW}$. For maximum penetration, the highest value of active power is $16.29 \mathrm{~kW}$ at $5: 45 \mathrm{pm}$. The difference between these values is almost $38 \%$.

Figure 9 presents the heat map for phase B under maximum penetration of DG-PV-ESS. There is a predominance of the nominal voltage magnitude during most of the time on all buses, which is also the highest voltage value. However, in the morning, the voltages present values equal or even lower than in previous situations. This results from the increased demand for active power as the ESSs will be in their inactive state at the beginning of the period. In the area that represents peak hours, a darkening is observed. However, it is less than the one observed in Figure 7 , which represents an improvement in voltage profiles. The lowest value presented at the maximum penetration of $144 \%$ is 0.963 p.u..

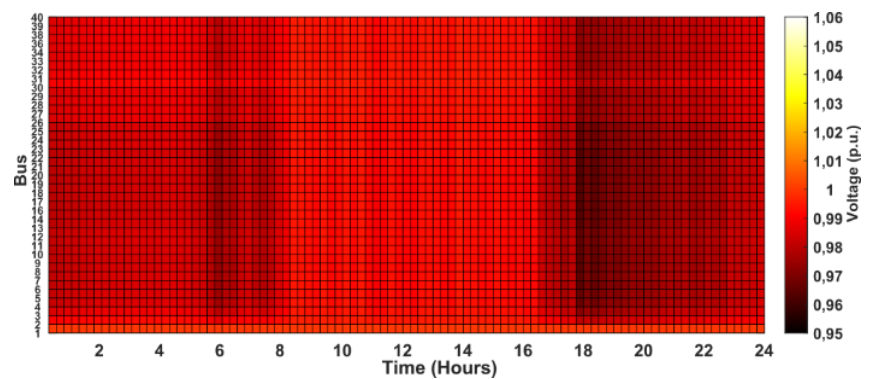

Figure 9 - Bus voltage variations in phase $B$ over the day with $144 \%$ DG-PV-BESS penetration

For comparison purposes with previous cases of local energy storage, the analyses of network's behaviour with only one larger ESS responsible for supplying all connected units is carried out. This study is performed only for the maximum DG-PV penetration, as this configuration is only justified for situations with large power injection at the network entrance.

To manage the ESS's charge and discharge, a native OpenDSS control device is applied, the Storage Controller (SC) proposed in [16]. In this particular case, the Peak Shave mode is used, which works similarly to the control outlined in section 3 . A minimum limit $(0 \mathrm{~kW})$ and a maximum limit $(7 \mathrm{~kW})$ are defined for the three-phase power monitored by the controller at the secondary transformer's side.

Figure 10 shows the active three-phase and single-phase powers in the ESS connection point, as well as in the transformer's secondary. The most pertinent aspect is that the ESS, together with the control adopted, successfully manages to cut all the surplus of active power exported through the transformer and reduce all consumption that exceeds $7 \mathrm{~kW}$ during peak hours. It can also be seen in the total power of the ESS, which takes the exact shape of the DG-PV during its charging period. The powers measured in the three phases of the ESS are the same throughout time. Such information is essential to understand the power profiles per phase in the transformer, shown in Figure 11.

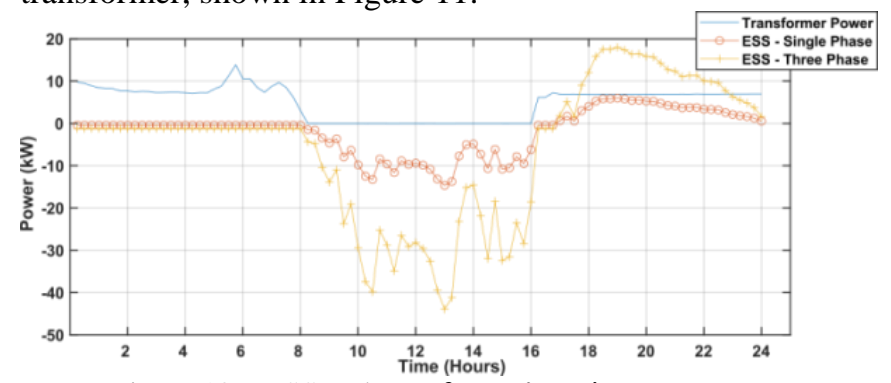

Figure 10 - ESS and transformer's active powers.

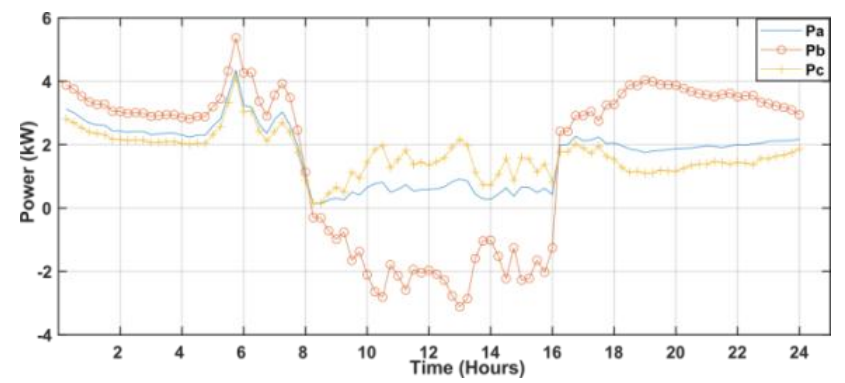

Figure 11 - Transformer's active power per phase.

The SC uses the measurements and limits related to the three-phase power, the algebraic sum of the three singlephase. Therefore, the controller operates by making the ESS to absorb or to inject the exact amount of active power in the three phases so that the total demand curve in the transformer only assumes values between 0 (zero) and $7 \mathrm{~kW}$. In turn, the transformer's powers in each phase always assume different values. It implies that for the minimum total power, imposed to zero by the control, and phase $\mathrm{B}$ must inject power while phases $\mathrm{A}$ and $\mathrm{C}$ present a positive demand. As noted in the previous sections, this difference can interfere with the phase voltages' unbalance and magnitudes.

Table I shows the resolution of line and transformer losses, the minimum and maximum voltage variations, and the maximum VUF\% phase. This information is presented for the network scenario in its original configuration and maximum DER penetrations.

Table I. - Impact indicators on network operation.

\begin{tabular}{|c|c|c|c|c|c|}
\hline \multicolumn{2}{|c|}{ Indicators } & $\begin{array}{c}\text { Original } \\
(0 \%)\end{array}$ & $\begin{array}{c}\text { DG-PV } \\
(144 \%)\end{array}$ & $\begin{array}{c}\text { DG-PV- } \\
\text { ESS } \\
(144 \%)\end{array}$ & $\begin{array}{c}\text { Concentred } \\
\text { PV-ESS } \\
(144 \%)\end{array}$ \\
\hline \multirow{3}{*}{$\begin{array}{c}\text { Variation } \\
(\%)\end{array}$} & $V_{\min }$ & -4.94 & -4.88 & -3.69 & -3.54 \\
\cline { 2 - 6 } & $V_{\max }$ & 0 & 3.92 & 0 & 1.15 \\
\cline { 2 - 6 } & VUF $F_{\max }$ & 0.44 & 0.57 & 0.29 & 0.69 \\
\hline \multirow{2}{*}{$\begin{array}{c}\text { Losses } \\
(\mathrm{kW})\end{array}$} & Line & 9.12 & 16.59 & 4.8 & 12.79 \\
\cline { 2 - 6 } & Tran. & 8.13 & 14.82 & 4.28 & 3.86 \\
\cline { 2 - 6 } & Total & 17.26 & 31.42 & 9.08 & 16.65 \\
\hline
\end{tabular}


With the DG-PV-ESS proposed system's employment, it is possible to reduce all impacts caused by the traditional DG-PV profile, improving the PQ indices and to still minimize lines and the distribution transformer losses. It is important to remember that the self-consumption of locally generated energy increases, representing an improvement in the use of the renewable sources and a decrease in consumer energy cost.

Finally, the use of a concentrated ESS proved to be effective. A good improvement is obtained regarding voltage drops. However, it is not as effective as DG-PVESS systems in containing voltage rises. It also shows an VUF\% increase. However, it remains well below the $3 \%$ limit required by ANEEL. Total losses also increase, assuming a value of $16.65 \mathrm{~kW}$, which is slightly lower than the original $17.26 \mathrm{~kW}$. The most exciting point in this sense is that there is an increase of losses in the lines conductors while reducing transformer losses. This decrease is probably due to the significant reduction in the active power demand from the primary network, thus restricting the transformer's energy dissipation. The increase of power dissipation in the conductors, on the other hand, happens because there is a bidirectional energy flow to the single storage. In this configuration, there will be a power flow throughout the day charging the ESS. There will be an inversion of it, discharging the accumulated reserve and feeding the loads. What allows these energy exchanges are, precisely, the distribution lines, and for that reason, there will be a greater dissipation on them.

\section{Conclusion}

The integration of ESSs, both distributed and concentrated, improves a high penetration grid-connected DG-PV. Both the maximum and the minimum bus voltage variations measured are reduced. There is also a considerable easing of total losses, which increase with the usual DG-PVs penetration. There are also improvements in this regard in comparison to the original network configuration.

Improvements are also observed in the VUF\% since voltage variations are reduced. However, in the concentrated ESS case, since it is integrated into the system by a frequency inverter (and all DERs), its output power is always the same for the three phases. As the consumption of the residential units connected to the network is not balanced between the conductors, the ESS control cannot match these values. Therefore, there will still be a considerable reverse active power flow in the transformer and the VUF\% deterioration in some points.

\section{Acknowledgements}

The authors would like to thank the Electric Energy Systems Laboratory (LSEE) of the São Carlos School of Engineering (EESC - USP) for the infrastructure provided, as well as the Coordination for the Improvement of Higher Education Personnel (CAPES) and the National Council Scientific and Technological Development (CNPq), for the financial support made available for this research.

\section{References}

[1] IRENA, "Renewable capacity statistics 2020," 2020.

[2] ABSOLAR, "Infográfico Energia Solar Fotovoltaica no Brasil | ABSOLAR." 2020.

[3] J. L. Paixão, A. R. Abaide, J. Rigodanzo, and J. P. Sausen, "Impact Study of the Photovoltaic Generation Entrance in a Dealership Network," in 2018 IEEE PES Transmission Distribution Conference and Exhibition Latin America (T D-LA), 2018, pp. 1-5.

[4] R. A. Walling, R. Saint, R. C. Dugan, J. Burke, and L. A. Kojovic, "Summary of Distributed Resources Impact on Power Delivery Systems," \{IEEE\} Trans. Power Deliv., vol. 23, no. 3, pp. 1636-1644, Jul. 2008, doi: 10.1109/tpwrd.2007.909115.

[5] M. M. Haque and P. Wolfs, "A review of high $\{\mathrm{PV}\}$ penetrations in $\{\mathrm{LV}\}$ distribution networks: Present status, impacts and mitigation measures," Renew. Sustain. Energy Rev., vol. 62, pp. 1195-1208, 2016, doi: 10.1016/j.rser.2016.04.025.

[6] A. R. Sparacino, G. F. Reed, R. J. Kerestes, B. M. Grainger, and Z. T. Smith, "Survey of battery energy storage systems and modeling techniques," 2012, doi: 10.1109/pesgm.2012.6345071.

[7] K. M. Muttqi, J. Aghaei, M. Askarpour, and V. Ganapathy, "Minimizing the steady-state impediments to solar photovoltaics," Renew. Sustain. Energy Rev., vol. 79, pp. 1329-1345, Nov. 2017, doi: 10.1016/j.rser.2017.05.065.

[8] R. Torquato, D. Salles, C. O. Pereira, P. C. M. Meira, and W. Freitas, "A Comprehensive Assessment of $\{P V\}$ Hosting Capacity on Low-Voltage Distribution Systems," \{IEEE\} Trans. Power Deliv., vol. 33, no. 2, pp. 1002-1012, 2018, doi:

10.1109/tpwrd.2018.2798707.

[9] ANEEL, "PRODIST - Módulo 8 - Qualidade da Energia Elétrica," Procedimentos Distrib. Energ. Elétrica no Sist. Elétrico Nac. - PRODIST, 2021.

[10] L. C. Brolin, F. B. B. Rolim, and F. C. L. Trindade, "Fast screening approach to estimate the hosting capacity of rooftop $\{\mathrm{PV}\}$ generators in electric power distribution systems," Int. Trans. Electr. Energy Syst., vol. 29, no. 3, p. e2740, 2018, doi: 10.1002/etep.2740.

[11] R. C. Dugan and A. Ballanti, "OpenDSS Manual," Train. Mater., no. March, 2016.

[12] P. Radatz, C. Rocha, W. Sunderman, M. Rylander, and J. Peppanen, "OpenDSS PVSystem and InvControl ElementModels." 2020.

[13] S. M. Ismael, S. H. E. A. Aleem, A. Y. Abdelaziz, and A. F. Zobaa, "State-of-the-art of hosting capacity in modern power systems with distributed generation," Renew. Energy, vol. 130, pp. 1002-1020, Jan. 2019, doi: 10.1016/j.renene.2018.07.008.

[14] C. Rocha and P. Radatz, "Elemento Storage do OpenDSS.” 2020.

[15] S. M. S. Danish, M. Ahmadi, M. S. S. Danish, P. Mandal, A. Yona, and T. Senjyu, "A coherent strategy for peak load shaving using energy storage systems," J. Energy Storage, vol. 32, p. 101823, 2020, doi: 10.1016/j.est.2020.101823.

[16] C. Rocha, J. Peppanen, P. Radatz, M. Rylander, and R. Dugan, "StorageController Element.” 2020. 